\title{
Comorbidity of personality disorder in obsessive- compulsive disorder: special emphases on the clinical significance
}

\author{
TianHong Zhang, ' Annabelle Chow, ${ }^{2}$ YingYing Tang, ' LiHua Xu, 'YunFei Dai, \\ KaiDa Jiang, ${ }^{1}$ JiJun Wang, ${ }^{*}$ and ZePing Xiao ${ }^{\text {* }}$
}

\footnotetext{
${ }^{1}$ Shanghai Mental Health Center, Shanghai Jiaotong University School of Medicine, Shanghai, PR China

${ }^{2}$ Department of Psychological Medicine, Changi General Hospital, Singapore
}

Obsessive-compulsive disorder (OCD) has been reclassified in the separate chapter obsessive-compulsive and related disorders (OCRDs) of the Diagnostic and Statistical Manual of Mental Disorders, Fifth Edition (DSM-V), indicating its distinction from other anxiety disorders for both phenomenology and etiology. However, the proposed alternative hybrid model for personality disorder (PD) was not accepted in the main body of the DSM-V. ${ }^{1}$ The PD category retains the DSM Fourth Edition (DSM-IV) 10 PDs and shifts to a single axis system. It could be predicted that the comorbidity rate between OCD and PD would be drop due to those changes that happened in DSM-5. We had previously reported that clinicians tend to neglect the possibility of diagnosing a patient with $\mathrm{PD}$ in China. ${ }^{2}$ This is especially true when the patient exhibits clear OC symptoms. The truth is that, in clinical routine practice, not only the OCD patients themselves, but also their friends and family members, have struggled long against the personality pathology along with the course of OCD. Also, although their ways of thinking and feeling are almost always stubborn, dogmatic, and inflexible, which related to PD definition as stability in the signs and symptoms, clinicians may not actively discriminate PD in OCD patients.

It is noticeable that the obsessive-compulsive personality disorder (OCPD), as defined by the DSM-V (orderliness, perfectionism, excessive attention to details, mental and interpersonal control, and at the cost of efficiency, flexibility, and openness), has long been of great interest to clinicians as bearing a close relationship with OCD. Although it had been widely debated across the DSM

* Address for correspondence: Zeping Xiao, MD, PhD, or Jijun Wang, MD, PhD, Shanghai Mental Health Center, 600 South Wanping Road, Shanghai 200030, PR China.

(Email: xiao_zeping@126.com; xiaozeping88@163.com; jijunwang27@163.com) working group regarding whether or not OCPD should be grouped with OCRDs, OCPD is better conceptualized as a disorder of personality for separating "personality characteristic" from "mental state" disorder. In many earlier studies, it was reported that about 50\%-80\% OCD patients met the criteria of OCPD (DSM, Third Edition; DSM-III), but in recent years, the rate was dropped to between $15 \%$ and $40 \%$ (DSM-IV). ${ }^{3}$ Due to those epidemiological surveys, which estimated that the comorbidity rate of OCPD in OCD was quite varied, neither persuasive evidence nor conclusion can be made regarding the etiological relationship between OCD and OCPD.

It is crucial to assess $\mathrm{PD}$ among patients with OCD; obviously, this will be beneficial to practicing clinicians. First, assessing PD features could be helpful in better predicting more severe OCD symptoms and poorer outcome of OCD. PD features also may indicate a vulnerability to difficulties in interpersonal activities and more functional deterioration in the future. ${ }^{4}$ A recent study has indicated that comorbid borderline PD has a significant negative longitudinal impact on the course and outcome of patients with OCD. The authors suggested that PD should be treated concurrently with OCD, as PD worsening significantly predicted OCD relapse. ${ }^{5}$ Second, considering the characteristics of the OCD course, which is well-known for its long-standing duration and ease of relapse, appropriate intervention strategies targeting PD at very beginning would improve the prognoses. For instance, patients with OCD accompanied by schizotypal PD, psychological support should be tailored to address the magical thinking, unusual beliefs, "just-right" feelings. Otherwise, if OCD is accompanied by schizoid PD, supporting approaches should address interpersonal difficulties, which may help to reduce social ineptness. ${ }^{6}$ Therefore, PD should receive full therapeutic attention alongside OCD. 

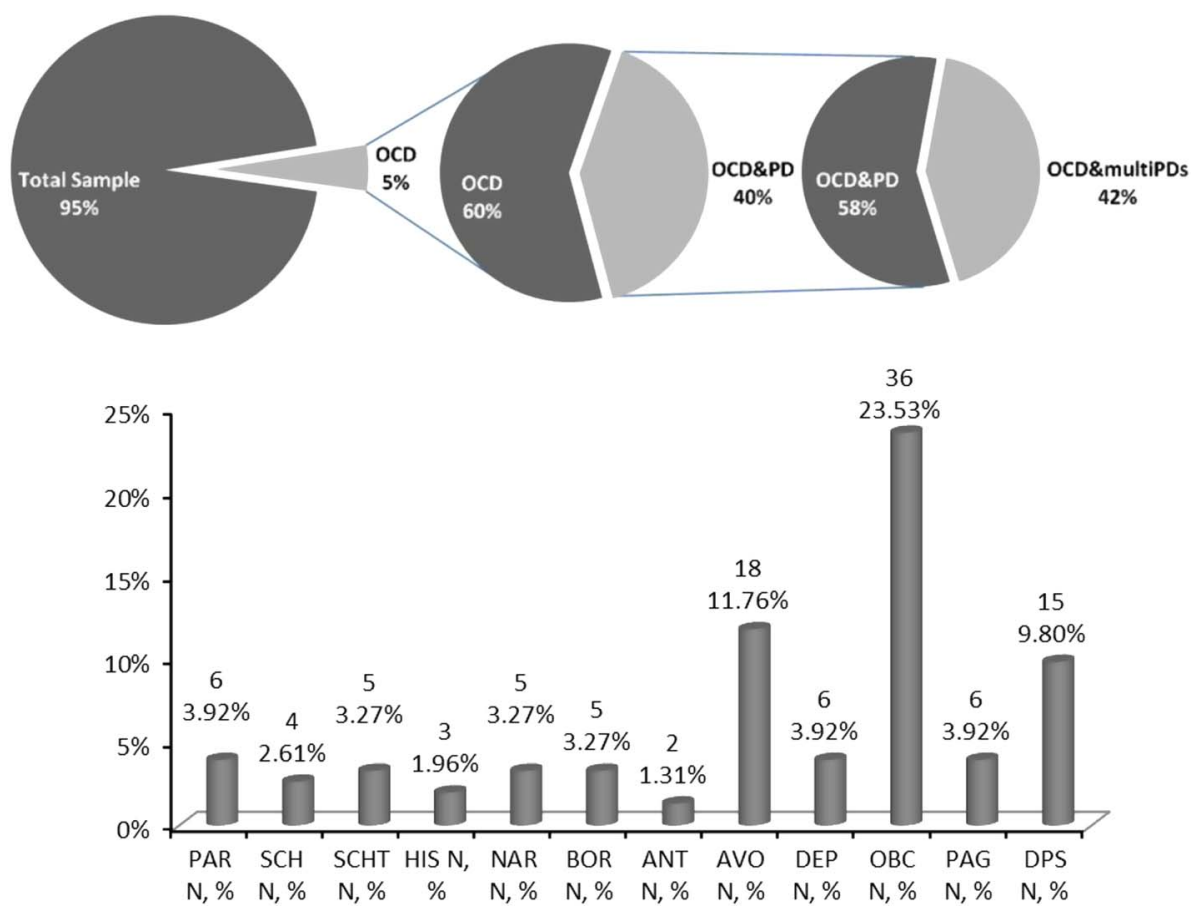

FIGURE 1. Diagnostic overlap among patients with OCD and DSM-IV PDs. Note: Paranoid PD (PAR); schizoid PD (SCH); schizotypal PD (SCHT); histrionic PD (HIS); narcissistic PD (NAR); borderline PD (BOR); antisocial PD (ANT); avoidant PD (AV0); dependent PD (DEP); obsessive-compulsive PD (OBC); passiveaggressive PD (PAG); depressive PD (DPS).

From an etiological point of view, the prevalence of PD comorbidity among patients with OCD implies that both share common phenomenological and neurobiological characteristics. Especially, a model linking OCPD to OCD may also act as one of the key etiological hypotheses on OCRD. ${ }^{3}$ Other than a cross-sectional investigation on $\mathrm{PD}$ assessment among OCD patients at a large scale, future research can consider following up with OCD patients diagnosed with PD to ascertain how the disorders are capable of acting on or influencing each other. Researchers should also test a few hypotheses in an effort to enhance our understanding of their interrelations ${ }^{7}$. The following are a few possibilities:

- According to the vulnerability hypothesis, certain PD may be predictive of subsequent onset or relapse of OCD.

- According to the scar hypothesis, OCRD and other spectrum diseases also may impact PD features.

- According to the spectrum hypothesis, related PD and OCD share a common underlying diathesis.

- According to the pathoplasty hypothesis, personality pathology impacts on symptomatology, disability, and outcomes in OCD.

Thus far, we have concluded that assessing PD among OCD patients would enable the improvement of therapeutic strategy and the development of appropriate risk factors tailored to OCD patients' clinical and functional outcomes. However, there are no reports on the prevalence of PD in Chinese patients with OCD in the existing literature. Even in the Chinese literature, the data regarding pathological personality in OCD are rare. Since we had conducted an epidemiologic survey on PD in our clinical settings in Shanghai, ${ }^{2,8-10}$ it would be valuable to present summarized data on comorbidity rates between PD and OCD. A random sample of 3402 subjects was randomly taken from the largest outpatient mental health clinic offering medication management and psychotherapy in China. These outpatients looking for professional help are from different parts of the country. Of the whole sample, 3075 subjects met enrollment criteria and were included in the study. A self-reported questionnaire and face-to-face interview were administered to gather information on DSM-IV PDs and OCD.

The sample comprised 153 participants $(5.0 \%$, $153 / 3075$ ) with a diagnosis of OCD. The frequency of PD among respondents with OCD was common; 66 outpatients with OCD $(43.1 \%, 66 / 153)$ met criteria for at least one type of DSM-IV PD [out of 12 types, including passive-aggressive PD (PAG) and depressive PD (DPS)]. There was a higher prevalence of Cluster-C (anxious PD) PDs (34.6\%, 53/153), followed by Cluster-B (dramatic, emotional or erratic) PDs $(9.8 \%, 15 / 153)$ and Cluster-A (odd and eccentric PD) PDs (13/153, 8.5\%). The most prevalent PD was OCPD (23.5\%, 36/153), followed by the avoidant PD (18/153, 11.8\%). Diagnostic overlap among DSM-IV PDs (including PAG and DPS) 
was quite common across all the categories. Of those 153 OCD patients, $38(57.6 \%, 38 / 153)$ were diagnosed with only 1 type of PD, and $28(42.4 \%, 28 / 66)$ were diagnosed with at least 2 types of PD (multi-PDs). (See Figure 1 for details.)

It is becoming clearer now that personality pathology and OCD are often closely related. Though there remains much work to do to determine either the diagnostic validity of PD or the psychological model that describes the interactivity between PD and psychopathology, it is necessary to put pressure on psychiatrists to pay more attention to personality traits when considering a therapeutic intervention for OCD patients. We highly suggest prioritizing treatment for OCD, whereas PD should be treated concurrently with OCD. A more in-depth model that relates personality traits to OCD should be the emphasis and direction of future research.

\section{Disclosures}

TianHong Zhang has the following disclosures:

Shanghai Mental Health Center, Employee, Salary, Research Grants

Shanghai Jiao Tong University Foundation

14JCRY04, YG2014MS40

Doctoral Innovation Fund Projects from Shanghai Jiao Tong University School of Medicine

BXJ 201345

National Key Clinical Disciplines at Shanghai Mental Health Center

OMA-MH, 2011-873

Shanghai Key Laboratory of Psychotic Disorders $13 \mathrm{dz} 2260500$

National Natural Science Foundation of China 81201043, 81171267, 81171280, 81261120410, 8136112

Shanghai Municipal Natural Science Foundation 12ZR1448400

NIH Fogarty International Center grant D43TW05809

Annabelle Chow has the following disclosure: Changi General Hospital, Employee, Salary

YingYing Tang has the following disclosure: Shanghai Mental Health Center, Employee, Salary, Research Grants
LiHua $\mathrm{Xu}$ has the following disclosure: Shanghai Mental Health Center, Employee, Salary, Research Grants

YunFei Dai has the following disclosure: Shanghai Mental Health Center, Employee, Salary, Research Grants

KaiDa Jiang has the following disclosure:

Shanghai Mental Health Center, Employee, Salary, Research Grants

JiJun Wang has the following disclosure:

Shanghai Mental Health Center, Employee, Salary, Research Grants

ZePing Xiao has the following disclosure

Shanghai Mental Health Center, Employee, Salary, Research Grants

\section{REFERENCES:}

1. Skodol AE, Morey LC, Bender DS, Oldham JM. The alternative DSM-5 model for personality disorders: a clinical application. Am J Psychiatry. 2015; 172(7): 606-613.

2. Zhang T, Wang L, Good MJ, et al. Prevalence of personality disorders using two diagnostic systems in psychiatric outpatients in Shanghai, China: a comparison of uni-axial and multi-axial formulation. Soc Psychiatry Psychiatr Epidemiol. 2012; 47(9): 1409-1417.

3. Fineberg NA, Sharma P, Sivakumaran T, Sahakian B, Chamberlain SR. Does obsessive-compulsive personality disorder belong within the obsessive-compulsive spectrum? CNS Spectr. 2007; 12(6): 467-482.

4. Huang LC, Hwang TJ, Huang GH, Hwu HG. Outcome of severe obsessive-compulsive disorder with schizotypal features: a pilot study. J Formos Med Assoc. 2011; 110(2): 85-92.

5. Keuroghlian AS, Gunderson JG, Pagano ME, et al. Interactions of borderline personality disorder and anxiety disorders over 10 years. J Clin Psychiatry. In press. DOI: 10.4088/JCP.14m09748.

6. Brakoulias V, Starcevic V, Berle D, et al. The clinical characteristics of obsessive compulsive disorder associated with high levels of schizotypy. Aust N Z J Psychiatry. 2014; 48(9): 852-860.

7. Andersen AM, Bienvenu OJ. Personality and psychopathology. Int Rev Psychiatry. 2011; 23(3): 234-247.

8. Zhang T, Chow A, Wang L, Dai Y, Xiao Z. Role of childhood traumatic experience in personality disorders in China. Compr Psychiatry. 2012; 53(6): 829-836

9. Zhang T, Good MJ, Good BJ, et al. Age and remission of personality pathology in the psychotic disorders compared to mood and/or anxiety disorders. Int J Psychiatry Med. 2012; 44(3): 241-255.

10. Zhang TH, Chow A, Wang LL, et al. Childhood maltreatment profile in a clinical population in China: a further analysis with existing data of an epidemiologic survey. Compr Psychiatry. 2013; 54(7): 856-864. 